\title{
Using imperfect advance demand information in ordering and rationing decisions
}

\author{
Tarkan Tan ${ }^{\mathrm{a}, *}$, Refik Güllü ${ }^{\mathrm{b}}$, Nesim Erkip $^{\mathrm{c}}$ \\ a School of Industrial Engineering, Eindhoven University of Technology, P.O. Box 513, 5600MB Eindhoven, The Netherlands \\ ${ }^{\mathrm{b}}$ Industrial Engineering Department, Bogazici University, 34342 Istanbul, Turkey \\ c Department of Industrial Engineering, Bilkent University, 06800 Ankara, Turkey
}

\section{A R T I C L E I N F O}

\section{Article history:}

Received 30 November 2007

Accepted 27 April 2009

Available online 19 May 2009

\section{Keywords:}

Inventory/production

Advance demand information

Customer reliability

Periodic review

Demand classes

Rationing

\begin{abstract}
A B S T R A C T
In this paper, we consider an inventory problem with two demand classes having different priorities. The appropriate policy of rationing the available stock, i.e. reserving some stock for meeting prospective future demand of preferred customers at the expense of deliberately losing some of the currently materialized demand of lower demand class(es), relies on the estimation of the future demand. Utilizing current signals on future demand, which we refer to as imperfect advance demand information (ADI), decreases uncertainty on future demand and may help to make better decisions on when to start rejecting lower class demand. We develop a model that incorporates imperfect ADI with inventory ordering (replenishment) decision and rationing available stock. In a two-period setting, we show some structural properties, solve the rationing problem, and propose solution methods based on Monte Carlo simulation for the ordering problem. We conduct numerical tests to measure the impact of system parameters on the expected value of imperfect ADI, and provide useful managerial insights.
\end{abstract}

(c) 2009 Elsevier B.V. All rights reserved.

\section{Introduction and related literature}

In this paper, we analyze a generic problem that incorporates imperfect advance demand information in inventory replenishment and rationing decisions. Consider the following example of a style goods manufacturer delivering shipments for two distinct markets (say, to local retailers and to overseas buyers), where local retail stores have priority over the overseas buyers. The manufacturer's planning horizon is divided into two periods with possibly different lengths. At the beginning of the first period, the manufacturer has pre-season order information from his customers in the form of soft

\footnotetext{
* Corresponding author. Tel.: +31402473950; fax: +31402465949.

E-mail addresses: t.tan@tue.nl (T. Tan), refik.gullu@boun.edu.tr (R. Güllü), nesim@bilkent.edu.tr (N. Erkip).
}

commitments that are subject to revisions within the terms of their mutual contracts. Based on this order information and the on-hand available inventory produced during the pre-season, the manufacturer makes a decision on how many additional goods to produce within the first period for availability in the second period. After the customer order information is transformed into hard order commitments, the manufacturer decides on how much of the overseas customers' demand to satisfy and how much stock to reserve for the second period, by also taking into account the order information for the second period. We analyze such quantity flexibility environments with different customer priorities and availability of order information.

The order information in the example above is a form of advance demand information (ADI), which is a term that refers to the information on future demand in general. If the customers place their orders prior to their 
requirements, this constitutes perfect $\mathrm{ADI}$. In many cases, however, the available information on future demand-or the information that can be collected and processed in rather easy and inexpensive ways particularly due to advances in information technologies-includes impurity and uncertainty. We refer to this kind of ADI, where there is an early indication of prospective future orders, as "imperfect ADI". A simple example is a company that uses sales representatives to market his products, in which case the collection of sales representatives' information as to the number of customers interested in a product provides an indication about the future sales of that product, hence it constitutes imperfect ADI. Other applications include internet retailing, vendor managed inventory (VMI) applications and collaborative planning, forecasting, and replenishment (CPFR) environments. In the remainder of the text-unless noted otherwise-we use the terms ADI and imperfect ADI interchangeably, since we consider the imperfect case in this paper.

The second important aspect of our paper is modeling customer differentiation. In the style goods manufacturer example above, local retailers may have priority over the overseas customers-or the other way around-for reasons such as higher sales volume or contractual agreements that guarantee some high service level. Customers are differentiated according to the importance assigned to not being able to meet their demand. Differentiated customer classes may not only have relative priority over each other, but they may also have different ADI structures.

In our problem environment, there are two demand classes (or customer classes). We refer to the preferred customer class as class- 1 and the other one as class- 2 . An appropriate policy to handle the problem of facing demand from different classes is to reserve some part of the stock for the use of higher priority customers only; this is known as (inventory) rationing policy. According to this policy, demands from both classes are met until the inventory level drops down to a critical rationing level, but if the inventory level is below critical rationing level, only class-1 demand is met. This results in backordering or losing some class-2 demand with the intention of avoiding (or decreasing the number of) probable class- 1 backorders or lost sales. Rationing policy is shown to be optimal for some problem environments under different demand classes (see, for example, Topkis, 1968; Ha, 1997b). However, an optimal rationing policy, which is the amount to be rationed and its dynamic relation with some other possible factors (time, lead times, remaining lead times, ADI, number and importance of demand classes, etc.) depends on the problem environment and it remains an open question in a general context.

While making the critical decision of starting to reject customers with an expectation of future demand from preferred customers, it may be very important to know more about future demand. Therefore, current signals on future demand could be utilized for making better decisions on when to start rejecting lower class demand. In this paper, we investigate the impact of using imperfect ADI in a production/inventory system when two distinguished demand classes exist. In particular, we analyze a single-item two-decision epoch problem with the objective of minimizing the expected total costs, under the assumption that unmet demand is lost. The questions that we attempt to answer are: What is the optimal way to allocate the inventory among the demand classes under imperfect ADI? How can the ordering policies be determined in such a case? How do the system parameters affect the value of imperfect ADI?

Our work relates to the existing body of the literature on two main aspects: modeling ADI in production/ inventory decisions and rationing of available inventory. In what follows, we first review the related literature on modeling ADI, and then discuss the literature on inventory rationing. We differ from the papers reviewed, mainly by taking ADI into account in rationing available inventory.

Hariharan and Zipkin (1995) showed that perfect ADI improves the performance of a continuous-time inventory system in the same way as a reduction in lead times. Gallego and Özer (2001) modeled perfect ADI through a vector of future demands and showed the optimality of a state-dependent order-up-to policy in a discrete-time setting. Dellaert and Melo (2003) dealt with the lot-sizing problem in a similar environment. Karaesmen et al. (2002) considered a capacitated problem under perfect ADI and stochastic lead times. They modeled the problem via a discrete time make-to-stock queue. We refer the reader to Karaesmen et al. (2003) for a literature survey and treatment of perfect ADI in production/inventory systems.

The literature on different forms of imperfect ADI has been increasing rapidly in the recent years. Treharne and Sox (2002) considered a problem where the demand in any given period arises from one of a finite collection of probability distributions. DeCroix and Mookerjee (1997) considered a periodic-review problem in which there is an option of purchasing demand information at the beginning of each period. Van Donselaar et al. (2001) investigated the effect of sharing imperfect ADI between the installers of a project and the manufacturers, in a projectbased supply chain. Thonemann (2002) elaborated further on a similar problem in which there is a single manufacturer and a number of installers. Zhu and Thonemann (2004) considered a problem that consists of a number of customers who may provide their demand forecasts. Benjaafar et al. (2007) considered a continuous review production-inventory system with imperfect ADI and updates. Liberopoulos and Koukoumialos (2008) modeled a two-customer-class system via simulation where the customers in the second class provide imperfect ADI. Tan et al. (2007) considered an imperfect ADI situation where information is modeled in a rather general way. All the above studies utilize ADI in their respective settings, propose inventory policies (in some cases optimal inventory policies), and show the benefits of imperfect ADI.

In the content of supply chain contracts, Tsay et al. (1999) referred to inventory rationing problems as extremely difficult and considered them as generally intractable. De Véricourt et al. (2002) stated that the rationing problem is often viewed as an operational decision rather than a strategic one, and hence disregarded in the contracts. According to their empirical 
results, however, inventory rationing turns out to be important. One of the pioneering studies that models different demand classes is by Veinott (1965). He considered a periodic review model in which each period is divided into smaller subperiods. Production or procurement decisions can only take place at the beginning of a (major) period. He introduced the concept of "critical levels" describing a possible critical level rationing policy without analyzing it. Topkis (1968) elaborated on this idea and proved that the optimal policy can be described by a base stock ordering amount in a period and a set of critical rationing levels in each subperiod. Another study (Evans, 1968) confirms the results of Topkis in an environment with two customer classes. They both assume zero lead time for ordering. The critical rationing levels in each subperiod depend on the remaining time until the end of the period. Nahmias and Demmy (1981) compared the effect of rationing on the fill rate against a traditional system with no rationing, for two demand classes. Cohen et al. (1988) considered a discrete time (s,S) inventory model with two demand classes, but without rationing. Moon and Kang (1998), Melchiors et al. (2000), Deshpande et al. (2003), and Teunter and Klein Haneveld (1999) are among the studies on rationing problems under continuous review, mostly based on the ideas of Nahmias and Demmy (1981). Kranenburg and Van Houtum (2007) developed accurate and efficient heuristics to find the critical levels and the base-stock level in an (S-1,S) lost sales model with multiple demand classes.

Under periodic review setting, Sobel and Zhang (2001) considered a model with two demand classes with the difference that class- 1 demand is deterministic and must be met whereas class- 2 demand is stochastic and can be backordered (without rationing). Frank et al. (2003) extended their analysis to the case where rationing of the stochastic demand is possible.

Another branch of rationing literature relies on queuing theory. Ha (1997b) modeled a rationing problem with several demand classes and lost sales as a single server make-to-stock queue, and showed the optimality of a set of monotone rationing levels with Poisson arrivals and exponential production times, i.e. in an $\mathrm{M} / \mathrm{M} / 1$ setting. Ha (1997a), Dekker et al. (2002), de Véricourt et al. (2001, 2002) are other relevant studies in the same line. Finally, Enders et al. (2008) considered a model where excess demand for class- 1 demand is lost and that for class- 2 demand is backlogged. The authors developed an exact evaluation procedure by modeling the problem as a level dependent quasi-birth-death process.

Note that our work relates weakly with the literature on dynamic pricing with inventory considerations (or similarly to revenue management), as a part of that literature focuses on market environments where there is no opportunity for inventory replenishment over the remaining part of the period or selling season (see Elmaghraby and Keskinocak, 2003 for a review). Under such an environment, the rationing policy reserves the available stock for the preferred customers, without considering a price change.

To our knowledge, our work is the first attempt to model imperfect ADI structure together with rationing and inventory decisions. During the revision of an earlier version of this article we came across a paper by Gayon et al. (2008) that considers a similar environment, but employs a different treatment. Gayon et al. (2008) considered Markovian customer arrival and supply processes that allow them to model the problem as a continuous time Markov decision process and showed the properties of the optimal policy. Our treatment does not require distributional restrictions and uses a discrete time model.

The contribution of this study can be summarized as follows:

(a) We present and analyze a model that incorporates the use of imperfect ADI in the presence of customer classes. In particular, we model a decision problem in which the rationing decision follows the ordering decision (with a time lag).

(b) We show the effect of using imperfect ADI on the rationing decision.

(c) We characterize the behavior of the optimal rationing policy under imperfect ADI. Additionally, we obtain useful structural properties of the problem posed.

(d) We present computational analysis that provides valuable managerial insight for the design and operation of such systems.

The rest of this paper is organized as follows. In Section 2 we discuss our problem environment and present the solution to the inventory rationing problem under imperfect ADI when two demand classes exist. Given the optimal solution to the rationing problem, we obtain the optimal order quantity in Section 3. We examine the value of information aspect of ADI on the rationing decision in Section 4. Finally, we present our conclusions and discuss possible extensions in Section 5.

\section{Modeling framework}

In this section we introduce the two-period decision framework. We define notation as need arises, but a list of most important notation is presented in Table 1 for convenience. We analyze the problem by considering a two-period model. Two streams of customers (high priority and low priority) generate independent stochastic demand in both periods. Class- 1 demand (the demand class with higher priority) is either immediately satisfied or lost. Class-2 demand is accumulated until the end of each period and unmet demand at the end of the period is lost. Each unit of lost demand from a class-1 customer incurs a cost of $b_{1}$ to the system, and that from a class- 2 customer incurs a cost of $b_{2}$, such that $b_{1}>b_{2}$. Each unit of unsold item incurs a cost of $h$ per period. One can also use salvage cost instead of $h$ at the end of the second period to take the end-of-horizon effect into consideration. Let $K_{n}$ be the random variable denoting the ADI that is available at the beginning of period $n$, whose realization is $k_{n}$, for $n=1,2$. Let $D_{n}^{i}\left(k_{n}\right)$ be the continuous random variable that denotes the demand from class- $i$ customers that occurs in period $n$, for $i=1,2$ and $n=1,2$, and let $d_{n}^{i}$ denote the 
Table 1

Relevant notation.

\begin{tabular}{|c|c|}
\hline$K_{n}:$ & $\begin{array}{l}\text { Random variable denoting the ADI that is available at the } \\
\text { beginning of period } n(n=1,2)\end{array}$ \\
\hline$k_{n}:$ & Realization of $K_{n}$ \\
\hline$D_{n}^{i}\left(k_{n}\right)$ & $\begin{array}{l}\text { Random variable denoting the demand from class- } i \text { customers } \\
\text { that occurs in period } n(i=1,2, n=1,2)\end{array}$ \\
\hline$D_{n}\left(k_{n}\right):$ & $\begin{array}{l}\text { Random variable denoting the total demand in period } \\
n(n=1,2)\end{array}$ \\
\hline$d_{n}^{i}:$ & Realization of $D_{n}^{i}\left(k_{n}\right)$ \\
\hline$d_{n}:$ & Realization of $D_{n}\left(k_{n}\right)$ \\
\hline$G_{n}^{i}\left(w \mid k_{n}\right)$ & Distribution function of $D_{n}^{i}\left(k_{n}\right)(i=1,2, n=1,2)$ \\
\hline$G_{n}\left(w \mid k_{n}\right):$ & Distribution function of $D_{n}\left(k_{n}\right)(n=1,2)$ \\
\hline$x:$ & Starting inventory level in the system \\
\hline Q: & Initial order quantity \\
\hline$y:$ & Inventory position at the beginning of the second period \\
\hline$b_{i}$ : & Stock-out cost per unit lost sale for demand class $i(i=1,2)$ \\
\hline$h:$ & Inventory holding cost per unit per period \\
\hline$\tilde{R}:$ & Critical rationing level \\
\hline$R:$ & $\begin{array}{l}\text { Reserve level, i.e. the net reserved amount at the end of the } \\
\text { first period }\end{array}$ \\
\hline
\end{tabular}

realization of $D_{n}^{i}\left(k_{n}\right)$. Note that we consider $k_{n}$ as all of the available ADI on both class- 1 and class- 2 demands. For instance, there can be separate information on each demand class, which may be correlated. We also note that $k_{n}$ is the information available at the beginning of period $n$ for the (total) demand that will be realized at the end of period $n$. For modeling purposes, we do not impose a particular relation between the available information and the demand realization. For instance, in the extreme case, $k_{n}$ may be the exact number of demands that will be realized (perfect ADI), or it may just be a parameter (such as the mean) of the demand distribution. In order to simplify the notation, we suppress $k_{n}$ and denote the random variable for demand as $D_{n}^{i}$. We define $D_{n}=D_{n}^{1}+$ $D_{n}^{2}$ to denote the total demand in period $n$, for $n=1,2$. The periods are not necessarily of the same duration.

There are two decision epochs in the planning horizon. Production (or procurement) lead time is one period. There is a starting inventory of $x$ on hand.

The following order of events takes place:

(A) At the beginning of the first period

(1) available on-hand inventory $x$, and the ADI for the first period $k_{1}$ are observed,

(2) a replenishment order of size $Q$, due to arrive at the beginning of the second period, is given.

(B) At the end of the first period

(1) the available stock $x$ is made available to meet $d_{1}^{1}$ as much as possible,

(2) $k_{2}$ is observed,

(3) rationing decision is made and $d_{1}^{2}$ is met to the extent allowed by the rationing decision,

(4) inventory related costs are incurred.

(C) At the beginning of the second period

(1) $Q$ is received,

(2) inventory level is raised to $y=R+Q$, where $R$ is the reserve level.
(D) At the end of the second period

(1) $d_{2}^{1}$ is met as much as possible, and $d_{2}^{2}$ is met to the extent of available inventory,

(2) inventory related costs are incurred.

Note that the information as to the future demand is available in the system at both decision epochs, namely $k_{1}$ at the point of ordering decision, and $k_{2}$ at the point of rationing decision. This information should be utilized to make better ordering and rationing decisions. We schematically describe the collection of ADI and realization of demands in Fig. 1.

The model of our paper fits better to products with a short life cycle, such as the style goods example described in Section 1. Demand signals for such products may be of crucial importance, especially if the possible number of replenishments is few. Our model considers such a product with two epochs of ordering. The first ordering decision that is made with little or no information on future demand (or future fashion) determines the initial inventory. As more information on demand is collected, the decisions (second replenishment, and rationing available inventory during the lead time of this second replenishment) are made by utilizing this information. In such environments, it is more likely that unmet demand is lost due to competitive market conditions.

While we discuss our model in detail and show some structural properties in the rest of this paper, the objective function can be summarized as ${ }^{1}$ :

$\min _{Q} E_{K_{2}, D_{1}^{1}, D_{1}^{2}}\left[\min _{y}\left\{T R C_{1}+E_{D_{2}^{1}, D_{2}^{2}}\left[T R C_{2}\right]\right\}\right]$

where $y$, as will be shown later, is an inventory level that can be interpreted as the rationing-adjusted order-up-to level when combined with $Q$ and demand realizations of the first period, and $T R C_{1}$ and $T R C_{2}$ are the total relevant costs for the system at the end of the first and the second period, respectively. In Sections 2.1 and 2.2 we analyze the rationing problem which is depicted as the inner minimization over $y$ in (1), and subsequently we analyze optimal selection of the initial order quantity $Q$ in Section 3.

We note that the inner minimization problem in (1) depends on the realization of $\left(K_{2}, D_{1}^{1}, D_{1}^{2}\right)$. Therefore, for each such realization, we have a corresponding optimal rationing level, and hence an optimal order-upto level. Due to the nature of the system (rationing) these optimal levels cannot be explicitly characterized. Hence, we either need to use numerical integration, or need to use a Monte Carlo-based method for the evaluation of the expected total cost. In this article we choose to apply an integrated approach where a Monte Carlo method based on the analytical findings obtained in Theorems $1-3$ is used.

\footnotetext{
${ }^{1}$ The subscripts of $E$ are the random variables over which the expectation is taken.
} 


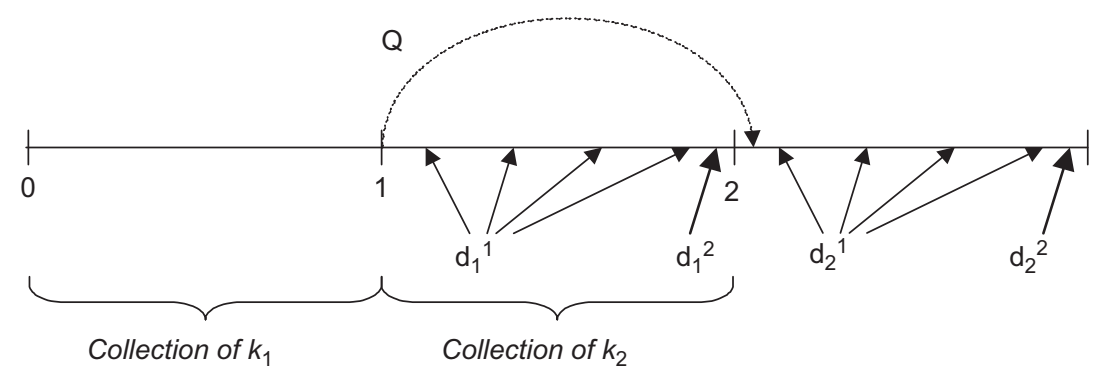

Fig. 1. Ordering and rationing problem with ADI.

\subsection{Rationing problem and derivation of the expected cost function}

We elaborate on the problem using a backward recursion and first handle the rationing problem at the end of the first period for a given set of parameters: $x, Q$, $d_{1}^{1}, d_{1}^{2}, k_{2}$. Note that $d_{1}^{1}, d_{1}^{2}$, and $k_{2}$ have already been observed at the beginning of the second period and that $d_{1}^{1}$ and $d_{1}^{2}$ depend on $k_{1}$, which was observed at the beginning of the first period.

Let $\tilde{R}$ be the non-negative critical rationing level such that if the inventory on hand after meeting class- 1 demand at the end of the first period is less than $\tilde{R}$, then all of $d_{1}^{2}$ is lost; otherwise, class- 2 demand is met as long as on hand inventory does not drop to a level less than $\tilde{R}$. Topkis (1968) showed that after making an ordering decision, the optimal policy to allocate the available stock to different classes of customers is of rationing type under possibly non-stationary demand distributions. For the problem environment that we consider, the existence of ADI leads to non-stationary demand. Given the quantity $Q$ to be received at the beginning of the second period, one can show that the structure of the optimal policy is still of rationing type, but as we discuss below the rationing amount is affected by $Q$ and other parameters.

We note that the problem has a trivial solution if $d_{1}^{1} \geq x$, as in this case all of the initial stock will be used to satisfy class- 1 customers.

Although it is possible to formulate the cost terms and hence the expected cost by making use of $\tilde{R}$, the formulation gets rather complicated then. Therefore we consider another variable, $R$, which is the inventory level right after meeting first period demand. Note that $R$ is related to $\tilde{R}$ in the following way:

$R= \begin{cases}0 & \text { if } x<d_{1}^{1}, \\ x-d_{1}^{1} & \text { if } x-\tilde{R} \leq d_{1}^{1} \leq x, \\ \tilde{R} & \text { if } x-\tilde{R}-d_{1}^{2} \leq d_{1}^{1}<x-\tilde{R}, \\ x-d_{1} & \text { if } d_{1}^{1} \leq x-\tilde{R}-d_{1}^{2} .\end{cases}$

$R$ is the net reserved amount at the end of the first period, either due to deliberate rationing or due to excessive or insufficient demand. Therefore, we refer to $R$ as the "reserve level". The relation defined in (2) translates into the following:

$$
\begin{aligned}
R & =\max \left\{\min \left\{\tilde{R},\left[x-d_{1}^{1}\right]^{+}\right\},\left[x-d_{1}\right]^{+}\right\} \\
& =\min \left\{\left(x-d_{1}^{1}\right)^{+}, \tilde{R}\right\}+\left(x-d_{1}-\tilde{R}\right)^{+} .
\end{aligned}
$$

We refer to the optimal value of $R$ as $R^{*}$, which is a function of $x, Q, d_{1}^{1}, d_{1}^{2}, k_{2}$, distribution functions of period2 demands, and cost parameters $b_{1}, b_{2}$, and $h$, as we derive in what follows.

Upon arrival of $Q$ at the beginning of the second period, the system adjusts its inventory level, which equals inventory position at that point, to

$y=Q+R$.

But since $Q$ is known at this point, finding the optimal value of $R$ is equivalent to finding the optimal value of $y$. Therefore, the rationing problem reduces to a modified newsboy problem with the cost function as derived below.

Inventory and lost sales costs at the end of the first period, $T R C_{1}$, can be stated as

$T R C_{1}=b_{1}\left(d_{1}^{1}-x\right)^{+}+b_{2}\left[R-\left(\left(x-d_{1}^{1}\right)^{+}-d_{1}^{2}\right)\right]+h R$.

Note that there is no stochastic term in $T R C_{1}$. We can substitute (4) in (5) to obtain

$T R C_{1}=b_{1}\left(d_{1}^{1}-x\right)^{+}+b_{2}\left[(y-Q)-\left(\left(x-d_{1}^{1}\right)^{+}-d_{1}^{2}\right)\right]+h(y-Q)$.

Inventory and lost sales costs at the end of the second period, $T R C_{2}$, can be stated as

$T R C_{2}=b_{1}\left(D_{2}^{1}-y\right)^{+}+b_{2}\left[D_{2}^{2}-\left(y-D_{2}^{1}\right)^{+}\right]^{+}+h\left(y-D_{2}\right)^{+}$,

which simplifies into

$T R C_{2}=\left(b_{1}-b_{2}\right)\left(D_{2}^{1}-y\right)^{+}+b_{2}\left(D_{2}-y\right)^{+}+h\left(y-D_{2}\right)^{+}$.

Combining (6) and (8), the inventory rationing problem at the end of the first period can be formulated $\mathrm{as}^{2}$

$\underset{y}{\operatorname{Minimize}} \quad E\left[T R C \mid k_{2}, d_{1}^{1}, d_{1}^{2}\right]=T R C_{1}+E\left[T R C_{2} \mid k_{2}, d_{1}^{1}, d_{1}^{2}\right]$

subject to $Q+\left(x-d_{1}\right)^{+} \leq y \leq Q+\left(x-d_{1}^{1}\right)^{+}$,

${ }^{2}$ We use the notation "E[TRC $\left.\mid k_{2}, d_{1}^{1}, d_{1}^{2}\right]$ " to refer to $E\left[T R C_{2} \mid K_{2}=\right.$ $k_{2}, D_{1}^{1}=d_{1}^{1}, D_{1}^{2}=d_{1}^{2}$, and similar notation for the conditional part whenever there is no ambiguity. 
where $T R C_{1}$ and $T R C_{2}$ are as defined in Eqs. (6) and (8), respectively, and both depend on $y$ and $Q$. In our notation, we suppress this dependency for brevity.

The constraints in (9) define the lower and upper bounds on the inventory level upon arrival of $Q$ at the beginning of the second period, respectively. Eq. (3) reveals that $R \geq\left(x-d_{1}\right)^{+}$and $R \leq\left(x-d_{1}^{1}\right)^{+}$. In other words, the minimum amount that must be reserved is what remains after meeting all of the demand in the first period, and the maximum amount that can be reserved is what remains after meeting class-1 demand in the first period, as discussed after defining $R$. Since $y=Q+R$, the constraints in (9) follow.

We refer to $E\left[T R C \mid k_{2}, d_{1}^{1}, d_{1}^{2}\right]$ as the "expected total conditional cost" (ETCC) and for ease of notation we drop $d_{1}^{1}, d_{1}^{2}$ terms in $E\left[T R C_{2} \mid k_{2}, d_{1}^{1}, d_{1}^{2}\right]$. Consequently,

$E T C C=T R C_{1}+E\left[T R C_{2} \mid k_{2}\right]$.

In particular,

$$
\begin{aligned}
E\left[T R C_{2} \mid k_{2}\right]= & \left(b_{1}-b_{2}\right) \int_{y}^{\infty}(w-y) d G_{2}^{1}\left(w \mid k_{2}\right) \\
& +\left(b_{2}+h\right) \int_{y}^{\infty}(w-y) d G_{2}\left(w \mid k_{2}\right)+h y-h E\left[D_{2} \mid k_{2}\right],
\end{aligned}
$$

where $G_{2}^{1}\left(w \mid k_{2}\right)$ and $G_{2}\left(w \mid k_{2}\right)$ are the distribution functions of $D_{2}^{1}$ and $D_{2}$, respectively. We note that while the demand may be discrete, for ease of exposition we assume that demand distributions are continuous and $E\left[T R C_{2} \mid k_{2}\right]$ is twice differentiable.

\subsection{Properties of the expected total conditional cost} function and the optimal reserve level

We first state the following theorem, which is used for solving the optimization problem presented in (9).

Theorem 1. ETCC is convex in $y$, and hence in $R$, for a given $Q \geq 0$ and for all $k_{2}, d_{1}^{1}$, and $d_{1}^{2}$.

Proof. $E\left[T R C_{2} \mid k_{2}\right]$ is convex in $y$ for all $k_{2}, d_{1}^{1}$, and $d_{1}^{2}$ since $T R C_{2}$ can be considered as the sum of two newsboy type functions (one with costs $b_{2}$ and $h$, and distribution $G_{2}$, and the other with costs $b_{1}-b_{2}$ and 0 , and distribution $G_{2}^{1}$ ), and $T R C_{1}$ is linear in $y$. Therefore, their sum is convex in $y$ for all $k_{2}, d_{1}^{1}$, and $d_{1}^{2}$. (See, e.g., Heyman and Sobel, 1984.) The same argument holds for $R$ for any given $Q \geq 0$, since $y=Q+R$.

One can find the optimal reserve level at the end of the first period as stated in the following theorem.

Theorem 2. The optimal reserve level at the end of the first period is

$R^{*}=\operatorname{Max}\left\{\operatorname{Min}\left\{\left(y^{*}-Q\right),\left(x-d_{1}^{1}\right)^{+}\right\},\left(x-d_{1}\right)^{+}\right\}$,

where

$y^{*}=\min \left\{y \mid\left(b_{2}-b_{1}\right)\left(1-G_{2}^{1}\left(y \mid k_{2}\right)\right)+\left(b_{2}+h\right) G_{2}\left(y \mid k_{2}\right)+h=0\right\}$

is the ideal inventory position at the beginning of the second period.
Proof. The first order condition is sufficient to find $y^{*}$, the minimizer of ETCC, due to Theorem 1. Then,

$$
\begin{aligned}
\frac{d E T C C}{d y} & =\frac{d T R C_{1}}{d y}+\frac{d E\left[T R C_{2} \mid k_{2}\right]}{d y} \\
& =\left(b_{2}+h\right)+\left(b_{1}-b_{2}\right) G_{2}^{1}\left(y \mid k_{2}\right)+\left(b_{2}+h\right) G_{2}\left(y \mid k_{2}\right)-b_{1},
\end{aligned}
$$

which results in (11). Combining this with the boundary conditions that are defined in (9), we obtain (10). We also note that there exists a solution to (11), because $\lim _{y \rightarrow-\infty}(d E T C C / d y)=-b_{1}+b_{2}+h<0, \quad$ and $\lim _{y \rightarrow+\infty}$ $(d E T C C / d y)=b_{2}+2 h>0$.

Theorem 2 enables us to characterize the relation between optimal reserve level $R^{*}$ versus $Q$ and $x$, given that the rest of the system remains the same. We enumerate some of those characterizing properties that relate $R^{*}$ with $Q$ in Corollary 1 , and those with $x$ in Corollary 2 .

Corollary 1. The following properties hold.

1. $R^{*}$ is a non-increasing function of $Q$ and $\lim _{Q \rightarrow \infty} R^{*}=\left(x-d_{1}\right)^{+}$.

2. If $0 \leq Q<\left(y^{*}-\left(x-d_{1}^{1}\right)^{+}\right)^{+}$, then $R^{*}=\left(x-d_{1}^{1}\right)^{+}$

3. $\frac{d R^{*}}{d Q}= \begin{cases}0 & \text { if } Q<Q_{l} \text { or } \\ 1 & \text { if } Q_{l}<Q<Q_{u}\end{cases}$

where

$$
Q_{l}=\left(y^{*}-\left(x-d_{1}^{1}\right)^{+}\right)^{+}, \quad Q_{u}=\left(y^{*}-\left(x-d_{1}\right)^{+}\right)^{+} .
$$

Property 1 is self-explanatory. Property 2 states that all that can be reserved should be reserved if $Q$ is not sufficient to reach the ideal inventory position at the beginning of the second period. Finally, property 3 states that for insufficient or abundant $Q$, marginal change in $Q$ does not affect $R^{*}$, since $R^{*}$ is solely determined by the initial inventory and first period demand in that case. For any $Q$ in between, the system reacts to a unit increase in $Q$ by a unit decrease in $R^{*}$ to maintain the ideal inventory position at the beginning of the second period, which is feasible in this region.

Corollary 2. The following properties hold.

1. $R^{*}$ is a non-decreasing function of $x$, and $\lim _{x \rightarrow \infty} R^{*}=\infty$.

2. If $x \leq d_{1}^{1}$, then $R^{*}=0$

3. $\frac{d R^{*}}{d x}=\left\{\begin{array}{lll}0 & \text { if } x<x_{l} \text { or } & x_{m}<x<x_{u}, \\ 1 & \text { if } x_{l}<x<x_{m} & \text { or } x>x_{u}\end{array}\right.$,

where

$x_{l}=d_{1}^{1}, \quad x_{m}=d_{1}^{1}+\tilde{R}^{*} \quad$ and $\quad x_{u}=d_{1}+\tilde{R}^{*}$.

Properties 1 and 2 are self-explanatory. Property 3 translates into the following: if $x$ is less than class- 1 demand, then a marginal change in $x$ does not affect $R^{*}$, since nothing is reserved anyway. If $x$ is more than class- 1 demand but not sufficient to reach the ideal inventory 

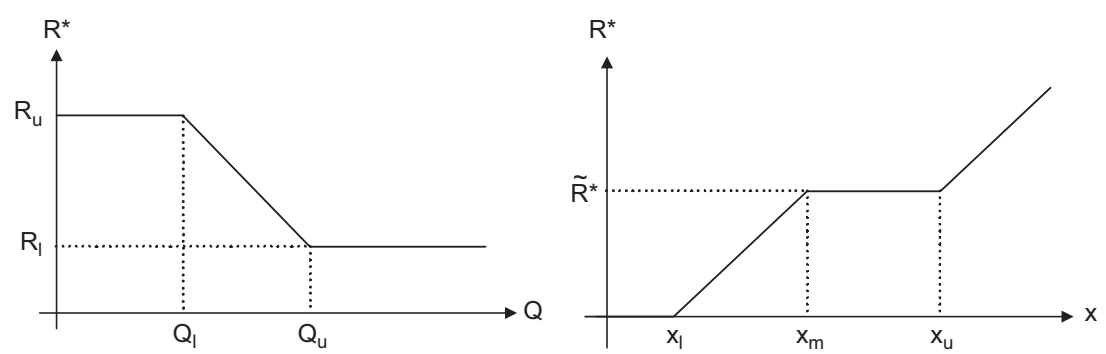

Fig. 2. Change of optimal reserve level versus order quantity and initial inventory.

position $y^{*}$, then the system will reserve all that it can. If $x$ is sufficient to reach $y^{*}$, then any $x$ in excess will be used to meet class-2 demand and hence will not be reserved, until all the demand is met. If $x$ is any larger than that, then it will have to be left over to the second period.

With the help of Corollaries 1 and 2, the relation between $R^{*}$ and $Q$ for a given $x$, and the relation between $R^{*}$ and $x$ for a given $Q$ can be illustrated as in Fig. 2, where $R_{u}=\max \left\{\left(\min \left\{y^{*},\left(x-d_{1}^{1}\right)^{+}\right\}\right),\left(x-d_{1}\right)^{+}\right\}$and $R_{l}=$ $\left(x-d_{1}\right)^{+}$. If $Q_{l}>0$, as in the illustration, then it turns out that $R_{u}=\left(x-d_{1}^{1}\right)^{+}$.

Finally, we consider the special case of perfect ADI on class- 1 demand, that is, when $d_{2}^{1}$ is known with certainty prior to the rationing decision at the end of the first period. For this case we obtain

$\frac{d E T C C}{d y}= \begin{cases}b_{2}+h-b_{1} & \text { if } y \leq d_{2}^{1}, \\ \left(b_{2}+h\right)\left(G_{2}^{2}\left(y-d_{2}^{1} \mid k_{2}\right)\right)+h & \text { if } y>d_{2}^{1} .\end{cases}$

This function is negative for the region $y \leq d_{2}^{1}$ since $b_{1}>b_{2}+h$ and positive for the region $y>d_{2}^{1}$ since $G_{2}^{2}$ is a distribution function. Consequently, $y^{*}=d_{2}^{1}$. This means that the ideal inventory level that the system would like to dedicate to the second period is the class- 1 demand that will be materialized in the second period, which in this case is known with certainty just before the rationing decision. Reserving below it would result in unmet class-1 demand in the second period, and reserving above it would result in reserving for class- 2 demand in the second period (i.e., possibly losing actual class-2 customers in the first period with an anticipation of class- 2 demand in the second period).

\section{Determination of the initial order quantity}

Now we proceed to the problem of deciding how much to order at the beginning of the first period that minimizes the expected total inventory related costs for both periods, $E[T R C(Q)]$, for a given $k_{1}$ and $x$. Note that $E[T R C(Q)]=$ $E_{K_{2}, D_{1}^{1}, D_{1}^{2}}\left[T R C_{1}+E\left[T R C_{2} \mid k_{2}, d_{1}^{1}, d_{1}^{2}\right]\right]$. We first state the following theorem.

Theorem 3. $E[T R C(Q)]$ is convex in $Q$ under the optimal reserve level policy that is defined in Theorem 2 .

Proof. Proof is provided in the Appendix.
Theorem 3 holds for any $R$, provided that $R$ is twice differentiable (piecewise) with respect to $Q$ and the second derivative is zero, as shown in the Appendix.

It is also interesting to observe that substituting (12) into (14) of the Appendix results in

$$
\left.\frac{d E\left[T R C_{2} \mid k_{2}\right]}{d Q}\right|_{R=R^{*}}= \begin{cases}\left(b_{1}-b_{2}\right) G_{2}^{1}\left(Q+R^{*} \mid k_{2}\right), & \\ +\left(b_{2}+h\right) G_{2}\left(Q+R^{*} \mid k_{2}\right) & \\ -b_{1} & \text { if } Q<Q_{l} \text { or } Q>Q_{u}, \\ 0 & \text { if } Q_{l}<Q<Q_{u}\end{cases}
$$

for the optimal reserve level. This result reveals that the marginal contribution of $Q$ to $E\left[T R C_{2} \mid k_{2}\right]$ within the limits $Q_{l}<Q<Q_{u}$ is zero. In other words, a decrease (or increase) of $Q^{*}$ by a marginal unit (as long as it is still in the limits mentioned) will result in the same $E\left[T R C_{2} \mid k_{2}\right]$, because the system will adjust itself to exactly the same inventory position by reserving a unit more (or less) for the second period.

We note that explicit evaluation of $E[T R C(Q)]$ is difficult, mainly due to the dependence of $R^{*}$ on $d_{1}^{1}, d_{1}^{2}$, and $k_{2}$. Therefore, we propose an approximate cost evaluation procedure (ACEP) based on Monte Carlo simulation in order to calculate approximate expected total inventory related costs. ACEP approximates the value of $E[T R C(Q)]$ for a given $Q \geq 0$, by generating a large number of realizations of $K_{2}, D_{1}^{1}$, and $D_{1}^{2}$, and taking the average of the optimal $E\left[T R C \mid k_{2}, d_{1}^{1}, d_{1}^{2}\right]$ values that are calculated for each realization. The remaining issue is to search for the optimal order quantity, $Q^{*}$, that minimizes $E[T R C(Q)]$. Theorem 3 enables the use of any search algorithm that is designed for convex functions.

Instead of approximating the expected total cost function $E[T R C(Q)]$, one can also approximate the derivative of $E[T R C(Q)]$ with respect to $Q$, again using Monte Carlo simulation. The idea is similar to that of ACEP, the difference being that the derivative of the optimal ETCC (see Eq. (15) in the Appendix) is computed this time for each set of realizations of $K_{2}, D_{1}^{1}$, and $D_{1}^{2}$. Finally, the optimal order quantity is the value of $Q$ for which $d E[T R C(Q)] / d Q$ is sufficiently close to zero.

\section{Value of information}

In this section we consider the value of information aspect of ADI on rationing decisions. We conduct numerical tests to find out under which circumstances a higher value of ADI exists. 


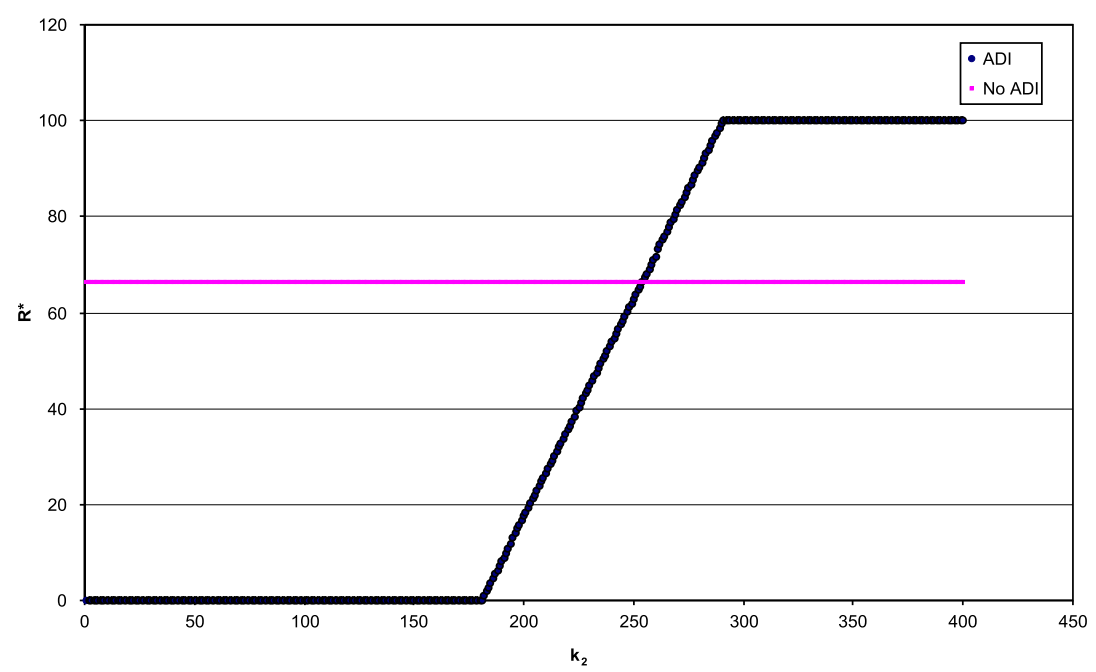

Fig. 3. Optimal reserve level versus ADI.

\subsection{Problem setting}

We note that we have assumed the demand distributions to be continuous in our analysis-while similar derivations could have been done for the discrete demand case as well-so we confine ourselves to continuous demand distributions in our numerical experiments. In the tests that we conduct, we consider a structure in which ADI is available on class- 1 customers only. The distribution of class-2 demand in the second period is taken to be normal with mean $\mu_{2}$ and variance $\sigma_{2}^{2}$, independent of class- 1 demand and ADI. We assume the conditional distribution of class- 1 demand in the second period to be normal with mean $k_{2} p$ and variance $k_{2} p(1-p)$, where $k_{2}$ represents the maximum class- 1 demand that may be materialized in the second period, and $p$ is a probability measure that represents the reliability of ADI. Our motivation for considering this distribution is the following information collection process: by the end of the first period, there may exist information on the potential number of customers in the system, $k_{2}$, each of which will generate a demand of size one with probability $p$ in the second period. In this case, the demand distribution upon realization of $k_{2}$ would be binomial with parameters $k_{2}$ and $p$, resulting in the mean $k_{2} p$ and variance $k_{2} p(1-p)$. Due to our continuous demand distribution assumption, we consider normal distribution with this mean and variance. The probability of demand realization, $p$, may be referred to as "customer reliability level", as well. While we assume homogeneity of customer reliability levels for simplification, this is not a restrictive assumption because non-homogeneous customer reliability levels can easily be accommodated in the model by segmenting the market according to the reliability levels as discussed by Tan et al. (2007). In the extreme case, a segment can be made up of a single customer.

We compare two policies for operating the system of concern in order to reveal the value of information:
ADI-case and NoADI-case. In both cases the decisions are made in an optimal manner, but only in the ADI-case is the system operated under advance demand information. For the sake of comparisons, the distribution of ADI is assumed to be known in the NoADI-case. We assume a normal distribution with mean $\mu_{K}$ and variance $\sigma_{K}^{2}$ for ADI. Our general approach is to obtain expected inventory-related costs for both of the cases and compare them.

Optimal reserve level under lack of $A D I$ : In order to find the optimal reserve level for the NoADI-case at the end of period 1, we use Theorem 2 as in the ADI-case, with the difference that the demand distributions are observed as $G_{2}^{1}(y)$ and $G_{2}(y)$, instead of $G_{2}^{1}\left(y \mid k_{2}\right)$ and $G_{2}\left(y \mid k_{2}\right)$, respectively, since ADI is not collected. The mean and the variance of $D_{2}^{1}$ can be evaluated by conditioning on $K_{2}$, as $E\left[D_{2}^{1}\right]=p \mu_{K}$ and $V\left[D_{2}^{1}\right]=p(1-p) \mu_{K}+p^{2} \sigma_{K}^{2}$. Thus, class- 1 demand distribution in the NoADI-case can be reflected by $D_{2}^{1} \sim N\left(p \mu_{K}, p(1-p) \mu_{K}+p^{2} \sigma_{K}^{2}\right)$, and the total demand in the second period under NoADI-case is normally distributed as well, since it is the sum of two independent normal distributions. Hence, $D_{2} \sim N\left(p \mu_{K}+\mu_{2}, p(1-p) \mu_{K}+\right.$ $p^{2} \sigma_{K}^{2}+\sigma_{2}^{2}$ ). As a result, there exists a unique reserve level for any given set of parameters at the end of the first period, as opposed to a variable reserve level that depends on $k_{2}$ in the ADI-case. Fig. 3 illustrates this point, where the change of optimal reserve level as a function of ADI size is plotted for ADI- and NoADI-cases under a certain set of parameters and a given $Q .^{3}$

\subsection{Experimentation}

Computation of the value of information: The system state at the instance of the rationing decision is defined by $x, d_{1}^{1}, d_{1}^{2}, Q$, and $k_{2}$, as well as cost parameters $b_{1}, b_{2}, h$, and

\footnotetext{
${ }^{3}$ While Fig. 3 gives the impression that the optimal reserve level is piecewise linear in $k_{2}$ in the ADI-case, it is actually (very slightly) nonlinear.
} 
distribution parameters $p, \mu_{K}, \mu_{2}, \sigma_{K}$, and $\sigma_{2}$. The approach we follow in order to reveal the value of information aspect of ADI in rationing decisions is to generate a large enough number of $k_{2}$ realizations, calculate corresponding optimal reserve levels and relevant costs, average the costs out, and compare. The performance measure that we consider is the expected percent penalty of not utilizing ADI, which we refer to as \% VoI. That is,

$\%$ VoI $=100 * \frac{E\left[T R C \mid d_{1}^{1}, d_{1}^{2}\right]_{\text {NoADI }}-E\left[T R C \mid d_{1}^{1}, d_{1}^{2}\right]_{A D I}}{E\left[T R C \mid d_{1}^{1}, d_{1}^{2}\right]_{A D I}}$

where $E\left[T R C \mid d_{1}^{1}, d_{1}^{2}\right]_{\text {NoADI }}$ and $E\left[T R C \mid d_{1}^{1}, d_{1}^{2}\right]_{A D I}$ denote the expected total relevant costs for the NoADI- and ADIcases, respectively, for given realizations of the first period demands. Since there is a fixed reserve level for the given input parameters in the NoADI-case independent of the value of $k_{2}, T R C_{1}$ in the NoADI-case does not change with $k_{2}$, therefore it suffices to compute it once. However, optimal reserve level depends on $k_{2}$ in the ADI-case, so $T R C_{1}$ is a function of $k_{2}$. Therefore we take the average of $T R C_{1}$ values conditioned on $k_{2}$ realizations in this case.

Description of the tests conducted: We conduct tests to examine the value of ADI on the rationing decision as a function of problem parameters. We first investigate which factors influence the value of ADI. Then we conduct tests for a range of values of some parameters in order not only to comprehend the effect of different values of those parameters on the value of information, but also to observe the sensitivity of the \% VoI.

We try different values of input parameters to investigate which values yield to increased \%VoI. We consider every combination of some fixed values of the input parameters, hence our experimental design follows a full-factorial fixed-effects model. The values of the input parameters (i.e., the levels of the factors) that we utilize in the experiment are provided in Table 2.

We fix $h=1$ and set the cost parameters according to two considerations: $b_{1} / b_{2}$ being close to 1 ( since $b_{1}>b_{2}+$ $h$ must hold, we set $b_{1} / b_{2}=1.3$ ) and being 10 ; and the trade-off between class-2 lost sales and inventory holding costs (i.e., $b_{2} /\left(b_{2}+h\right)$, which we refer to as " $b_{2} F R$ ", resembling approximate "fill rate" as if there are no demand classes) being 0.8 or 0.95 . The resulting sets of cost parameters are $\left(b_{1}, b_{2}\right)=(5.2,4),(40,4),(24.7,19)$, and $(190,19)$. The coefficient of variation, $C V$, is taken as common to $K_{2}$ distribution and $D_{2}^{2}$ distribution at the same time. That is, $\sigma_{K} / \mu_{K}=\sigma_{2} / \mu_{2}=C V$. We set $d_{1}^{1}=0$ in all sets, because $d_{1}^{1}$ is to be met from $x$ in all cases and if

\section{Table 2}

Values of input parameters.

\begin{tabular}{ll}
\hline$p$ & $0.1,0.5,0.9$ \\
$Q$ & $0,100,200$ \\
$x$ & 10,100 \\
$b_{1} / b_{2}$ & $1.3,10$ \\
$b_{2} F R$ & $0.8,0.95$ \\
$\mu_{K}$ & 20,200 \\
$\mu_{2}$ & 10,100 \\
$C V$ & $0.05,0.25$ \\
$d_{1}^{2}$ & 10,100
\end{tabular}

$d_{1}^{1}>x$ then there would be no inventory left to ration at the end of the first period, and the value of information would trivially be zero.

These values of parameters result in $2^{7} \times 3^{2}=1152$ sets. Since we are interested in revealing the characteristics of parameter sets that yield high \% VoI rather than obtaining confidence intervals on \%VoI for each set, we only evaluate point estimates. Nevertheless, we note that one might construct confidence intervals as well.

Discussion of the results: We conduct an analysis of variance (ANOVA) for this experiment on a model that includes all of the main and two-way interaction effects. The main effects that are significant on \% VoI at $95 \%$ confidence level (and their respective $p$-values ${ }^{4}$ ) are $\mu_{K}$ (0.001), $b_{1} / b_{2}$ (0.003), $\mu_{2}$ (0.004), $x$ (0.005), $d_{1}^{2}$ (0.017), $p$ (0.023), and $C V(0.028)$.

For this set of parameters, we deduce from main effects plots that there is a higher expected value of information on rationing decision when ADI signals a high demand, relative importance of class- 1 customers (i.e., $b_{1} / b_{2}$ ) is high, expected class- 2 demand of the second period is low, initial inventory level is high, class-2 demand of the first period is high, customer reliability level is high, and system variability (induced by $k_{2}$ and $D_{2}^{2}$ ) is high. These results are mostly in line with intuition, as we briefly discuss in what follows. It should be noted that none of the factors that lead to higher \% VoI can be effective only by themselves. For example, if there is no initial inventory, then the value of information will be zero, independent of all other factors, since there will be nothing to ration.

Observation 1: When class-1 is not significantly more important than class-2, and especially when both lost sales costs are relatively low, it does not pay off to collect $\mathrm{ADI}$, because the rationale for rationing (losing a class- 2 demand deliberately and also facing a holding cost to avoid a possible loss of class- 1 demand) diminishes.

Observation 2: Higher values of $\mu_{K}$ and $p$ stand for a higher expectation for class- 1 demand in the second period. In that case, reservation for the second period gets more critical, hence the value of information. We discuss this issue later in this section. We note that ADI is not only important when it signals a higher prospective demand, because when the ADI signals a lower prospective class-1 demand, it alerts the system accordingly, despite high expectation prior to ADI realization (i.e., when $E\left[K_{2}\right]=\mu_{K}$ is high but realized $k_{2}$ is relatively low). These arguments hold in a stronger sense when the variance of the ADI and the variance of class- 2 demand are higher, hence the variance of the second period demand is high.

Observation 3: A low level of initial inventory (relative to $d_{1}^{2}$ ) leaves less room for rationing, and therefore the expected value of information is less. A similar reasoning holds for the amount of class-2 demand in the first period, $d_{1}^{2}$ (relative to $x$ ).

Observation 4: When the expected class-2 demand of the second period is low, then the second period demand

\footnotetext{
4 " $p$-value" is the smallest level of significance that would lead to rejection of ANOVA null hypothesis, and it should not be confused with customer reliability level, $p$.
} 


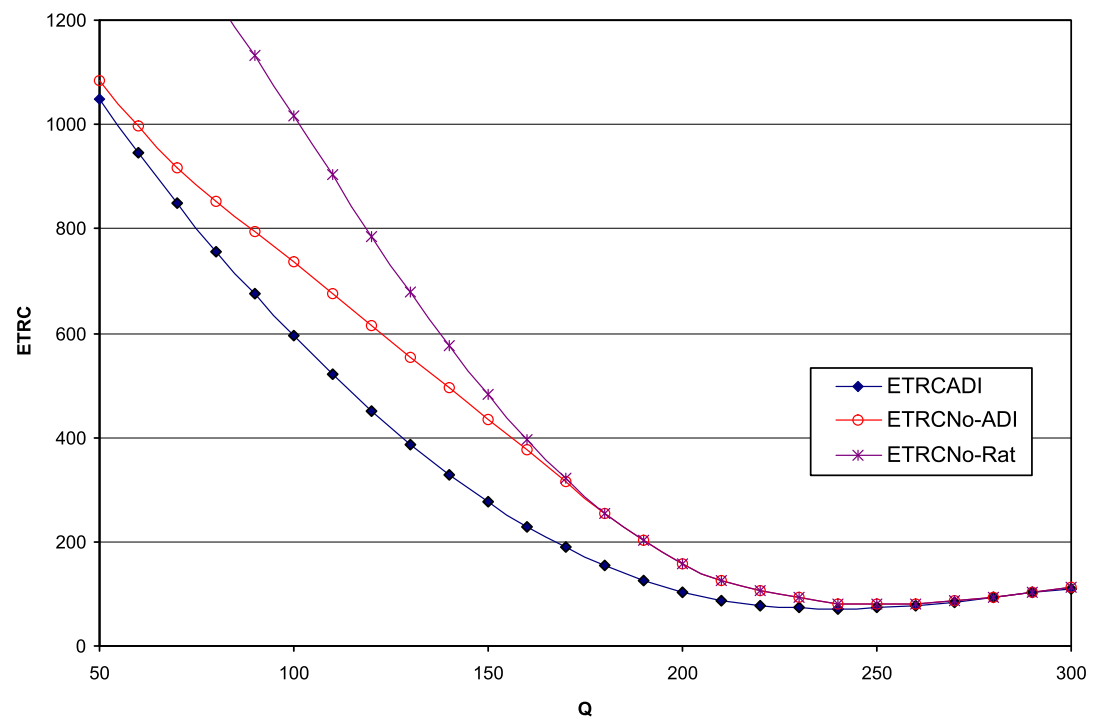

Fig. 4. Order quantity versus $E\left[T R C \mid d_{1}^{1}, d_{1}^{2}\right]$.

is mostly defined by class-1 demand. Therefore, ADI (which is on class-1 demand in this experiment) becomes more important.

The impact of $Q$ and $x$ on the \%VoI: The previous experiment does not make it very clear how the value of ADI changes as a function of $Q$. Nevertheless, in most of the results with higher \% VoI it appears that $Q$ is close to the expected class- 1 demand of the second period $\left(E\left[D_{2}^{1}\right]\right)$. Therefore, we conduct another ANOVA, this time with the factor $E\left[D_{2}^{1}\right]\left(=p \mu_{K}\right)$ instead of the factors $p$ and $\mu_{K}$. The main effects that are significant on $\% \mathrm{VoI}$ at $95 \%$ confidence level (and their respective $p$-values) are $E\left[D_{2}^{1}\right](0.000)$, $b_{1} / b_{2}$ (0.003), $\mu_{2}$ (0.004), $x$ (0.005), $d_{1}^{2}$ (0.016), and $C V$ (0.026). The most significant interaction effect is between $Q$ and $E\left[D_{2}^{1}\right]$ with a $p$-value of 0.000 .

We present the expected total cost figures, $E\left[T R C \mid d_{1}^{1}, d_{1}^{2}\right]$, for ADI- and NoADI-cases with $p=0.9$, $C V=0.25, \mu_{K}=200, \mu_{2}=10, x=100, d_{1}^{1}=0, d_{1}^{2}=100$, $b_{1}=12, b_{2}=5, h=1$ in Fig. 4 . Expected total costs when rationing is not allowed (ETRCNo-Rat) are also presented on the same graph for comparison purposes.

Observation 5: As Fig. 4 reveals, the penalty paid for not rationing compared to the optimal rationing policy is significant especially when the order quantity is smaller than optimal. Moreover, for order quantities that are much smaller than the optimal, the majority of the penalty is due to not rationing, whereas for the rest it is due to not employing imperfect ADI in the rationing decision. There are still considerable savings if rationing and imperfect ADI are used with the optimal ordering quantity.

We extend our analysis by examining the relation between $Q$ and \%VoI for different values of initial inventory level $x$, because $x$ and $Q$ interact in determining the reserve level. The values of the parameters in this set are $p=0.9, C V=0.25, \mu_{K}=200, \mu_{2}=100, d_{1}^{1}=0$, $d_{1}^{2}=100, b_{1}=190, b_{2}=19, h=1$. The \%VoI versus $Q$ for different values of $x \geq d_{1}$ are presented in Fig. 5, and those for $x \leq d_{1}$ are presented in Fig. 6. We note that when $x \geq$ $d_{1}$ (where $d_{1}=d_{1}^{2}$ in our current setting), the individual values of $x-d_{1}$ and $Q$ are irrelevant as long as their sum remains unchanged, because $x+Q-d_{1}$ is the lower bound on feasible $y$. Therefore, the absolute value of information is the same ${ }^{5}$ for the same $x+Q-d_{1}$ values, and \% VoI changes only due to their relative levels. For the case where $x \leq d_{1}$, \% VoI increases as $x$ increases for all values of $Q$.

Observation 6: In Figs. 4-6 we observe that the percentage VoI is relatively higher when $Q+\left(x-d_{1}\right)^{+}$is not much larger or smaller than the expected value of the second period class- 1 demand, and in particular when it is slightly higher than the expected value of the second period class- 1 demand. These constitute the cases where ADI (and the rationing decision as a function of ADI) becomes very valuable as a non-trivial decision is required. When $Q+\left(x-d_{1}\right)^{+}$is much larger or smaller than the expected second period class-1 demand, the relative importance of information disappears as there is not much to do. We note that the quantity rationed in excess of $E\left[D_{2}^{1}\right]$ acts like a safety stock, being a function of demand variability and cost parameters in the system.

The impact of $C V$ and $p$ on the \% VoI: We conduct further tests to investigate the behavior of \% VoI as customer reliability level $(p)$ and the coefficient of variation $(C V)$ change. The input parameters are $\mu_{K}=200, \mu_{2}=10, x=$ $100, d_{1}^{1}=0, d_{1}^{2}=100, b_{1}=12, b_{2}=5, h=1$. We let $p$ vary between 0.1 and 1 , and $C V$ between 0.05 and 0.25 . We also fix $Q$ at 100 . The results of these tests are summarized in Fig. 7.

\footnotetext{
${ }^{5}$ There are insignificant differences due to the simulation-based calculation of the expected costs.
} 


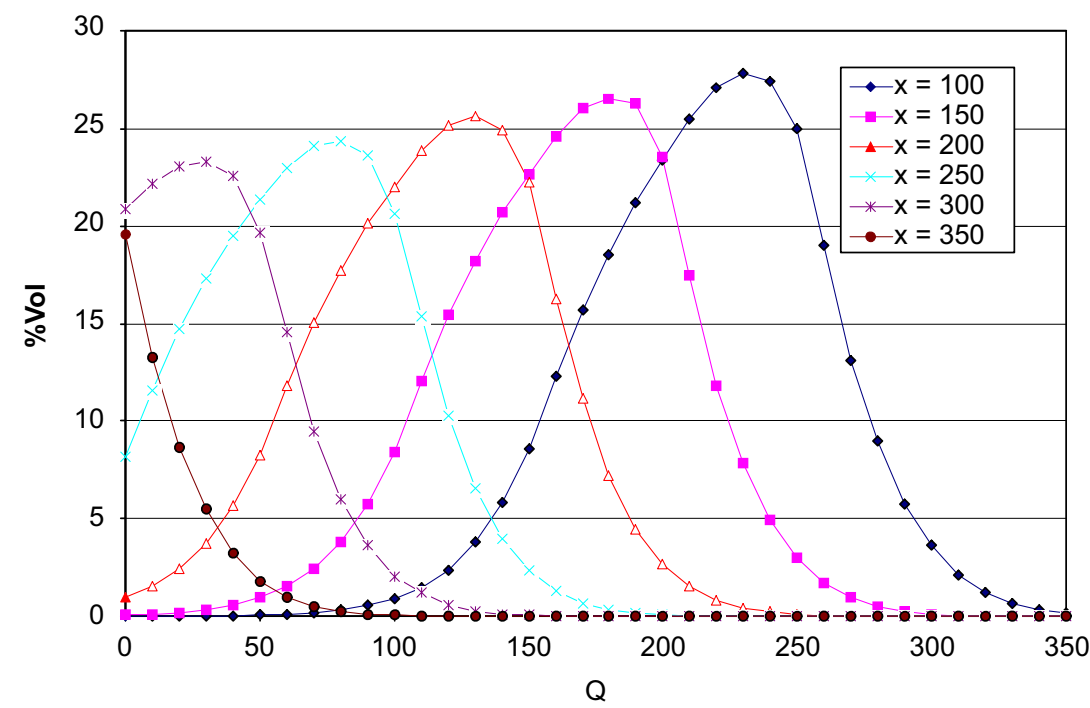

Fig. 5. Order quantity versus $\%$ VoI for $x \geq d_{1}$.

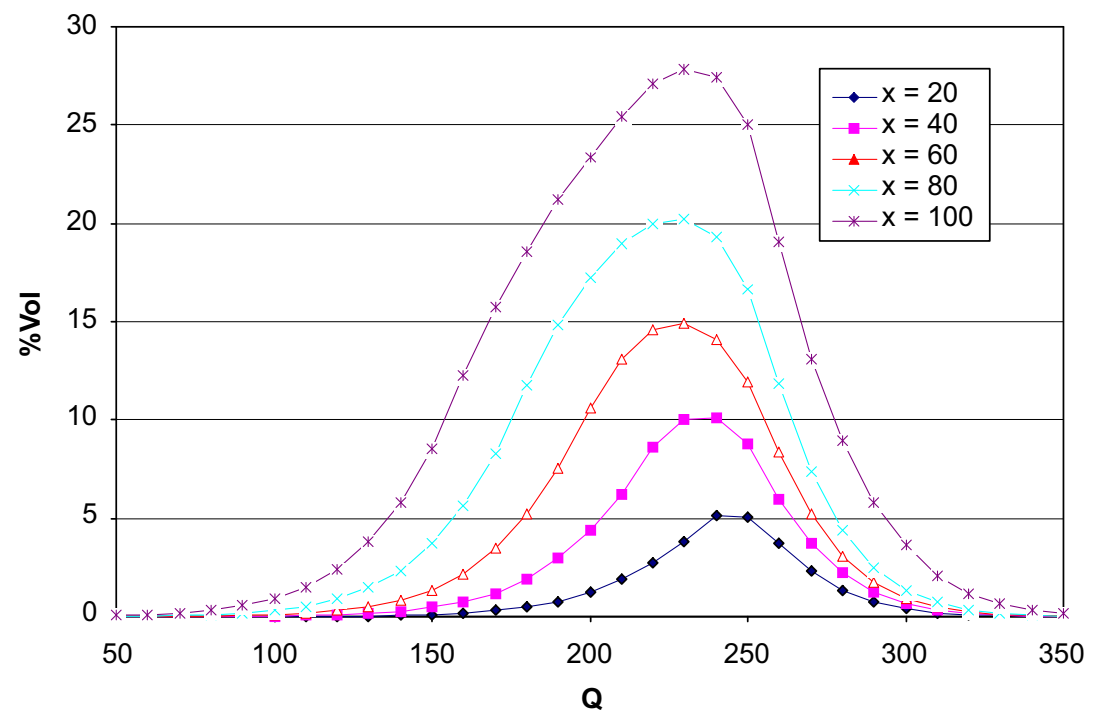

Fig. 6. Order quantity versus $\%$ VoI for $x \leq d_{1}$.

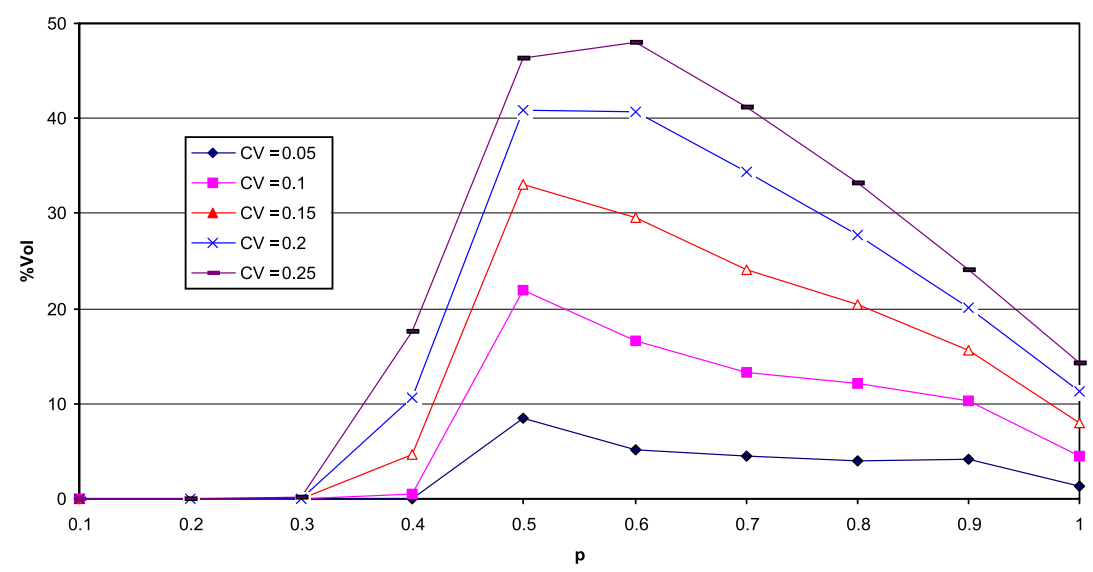

Fig. 7. Customer reliability level and coefficient of variation versus \%VoI. 
Observation 7: ADI increases as the coefficient of variation, hence the demand variance increases.

Observation 8: Other parameter levels are critical to draw any conclusion about $p$. \% VoI is zero for small values of $p$ in Fig. 7, because the current value of $Q=100$ covers for class-1 demand in the second period (with $E\left[D_{2}^{1}\right]<40$ for $p<0.2$ ) and the optimal policy is not to ration. On the other hand, the percent penalty of not utilizing ADI decreases for high values of $p$ in this setting, since the gap between the expected class-1 demand of the second period and $Q$ decreases the relative importance of information.

\section{Conclusions and future research}

The main motivation of employing imperfect advance demand information in an inventory/production system in general is that it can improve the performance of the system through decreasing uncertainty on future demand. When there are multiple demand classes of different priorities, then the appropriate policy of rationing the available inventory comes at the expense of possibly losing some of the currently materialized demand of lower demand classes. This delicate issue, after all, relies heavily on the estimation of the future demand. Therefore, utilizing current signals on future demand may be extremely important to make better decisions on when to start rejecting current demand.

In this paper we have developed a model that helps us investigate this problem in a simplified environment. We analyzed a system that is made up of one ordering and one rationing decision under two demand classes. Consequently, the rationing problem is solved analytically. A Monte Carlo simulation-based procedure is developed to evaluate the total expected inventory-related costs. Showing that expected cost function is convex in terms of order quantity, two methods are suggested for determining the optimal order level. A procedure is built to evaluate the expected value of imperfect ADI on the rationing decision. Numerical tests are conducted to measure the impact of system parameters on the expected value of imperfect ADI. Under the parameters we considered, the results of these tests revealed that imperfect ADI is more valuable when the demand variance is high, relative importance of class- 1 demand is high, there is sufficient class-2 demand at the first period and sufficient initial inventory to increase the flexibility to ration, and the order quantity plus the initial inventory in excess of the first period demand is close to the expected class- 1 demand of the next period plus some safety stock.

This study presents some issues of relevance with respect to the design and operation of such systems. The following is a relevant list of issues for managerial insight:

(a) Rationing is a difficult decision to apply in practice, as it may have an undesired influence on low-priority customer demand. On the other hand, if the return of such an action is significant then some incentives can be designed to prevent those undesirable influences.
We show that, in the environment we have described, the benefits can be sufficiently large, even though ADI is imperfect.

(b) The rationing decision is generally considered to be an operational decision. However, as shown in the computational analysis, especially if the system is operating with order quantities that are smaller than optimal, the penalty paid for not applying the optimal rationing policy can be significant. For order quantities that are much smaller than the optimal, the majority of the penalty is due to not rationing, whereas for the rest the majority of the penalty is due to not employing imperfect ADI in the rationing decision. There are still considerable savings if rationing and imperfect ADI are used with the optimal ordering quantity.

(c) Imperfect ADI and rationing are two important characteristics that will improve system performance where uncertainty, non-stationarity and long lead times are important features of the inventory system considered.

We note that although we have assumed no set-up cost in the analysis, our model can be extended to cover a positive set-up cost as well. Nevertheless, extending the model into a longer or infinite horizon multi-period structure is not as straightforward. Under a general lead time assumption, increased dimensionality becomes an important issue. A multi-period structure disallows considering the ordering and rationing decisions distinctly as we do in this paper, because optimality should be on both of those decisions in every period. A possible approach could be to pre-set a rationing policy as a function of ADI and then solve for $Q$, or vice versa. Another issue in the multi-period setting is the modeling of the ADI structure. While increasing the complexity of the problem, a possible solution or characterization could provide further insight. A natural extension is to handle the case of several customer classes, which may be necessary both for generalization purposes and for some possible applications. Instead of a single reserve level, there would be a reserve level for each class in this case, except for class-1.

\section{Acknowledgements}

The authors thank the guest editors for their efforts during the reviewing process. Tarkan Tan was at Middle East Technical University and Atilim University, Refik Gullu was at Middle East Technical University, and Nesim Erkip was at Middle East Technical University and Eindhoven University of Technology when parts of this research were carried out.

\section{Appendix A. Proof of Theorem 3}

We first show that $E\left[T R C \mid k_{2}, d_{1}^{1}, d_{1}^{2}\right]=T R C_{1}+$ $E\left[T R C_{2} \mid k_{2}, d_{1}^{1}, d_{1}^{2}\right]$ is convex in $Q$ for a given set of $k_{2}, d_{1}^{1}$, and $d_{1}^{2}$. The cost terms $T R C_{1}$ and $T R C_{2}$ are as stated in (6) and ( 8 ), and they can be stated in terms of $Q$ by noting that $y=Q+R$, with $R=R^{*}$ in case of optimal reserve policy. Hence, 


$$
\begin{aligned}
E\left[T R C_{2} \mid k_{2}, d_{1}^{1}, d_{1}^{2}\right]= & \left(b_{1}-b_{2}\right) \int_{Q+R}^{\infty}(w-(Q+R)) d G_{2}^{1}\left(w \mid k_{2}\right) \\
& +\left(b_{2}+h\right) \int_{Q+R}^{\infty}(w-(Q+R)) d G_{2}\left(w \mid k_{2}\right) \\
& +h(Q+R)-h E\left[D_{2} \mid k_{2}\right] .
\end{aligned}
$$

Note that $R$ is a function of $Q$, so the derivation of (13) requires derivation of $R$ with respect to $Q$ as well. (The derivative is given in (12) for $R=R^{*}$.) Let $R^{\prime}=d R / d Q$. Then,

$$
\begin{aligned}
\frac{d E\left[T R C_{2} \mid k_{2}, d_{1}^{1}, d_{1}^{2}\right]}{d Q}= & -\left(b_{1}-b_{2}\right) \int_{Q+R}^{\infty}\left(1+R^{\prime}\right) d G_{2}^{1}\left(w \mid k_{2}\right) \\
& -\left(b_{2}+h\right) \int_{Q+R}^{\infty}\left(1+R^{\prime}\right) d G_{2}\left(w \mid k_{2}\right)+h\left(1+R^{\prime}\right) \\
= & \left(1+R^{\prime}\right)\left[\left(b_{1}-b_{2}\right) G_{2}^{1}\left(Q+R \mid k_{2}\right)\right. \\
& \left.+\left(b_{2}+h\right) G_{2}\left(Q+R \mid k_{2}\right)-b_{1}\right]
\end{aligned}
$$

We also have $d T R C_{1} / d Q=\left(b_{2}+h\right) R^{\prime}$. Therefore,

$$
\begin{aligned}
\frac{d E\left[T R C \mid k_{2}, d_{1}^{1}, d_{1}^{2}\right]}{d Q}= & \left(1+R^{\prime}\right)\left[\left(b_{1}-b_{2}\right) G_{2}^{1}\left(Q+R \mid k_{2}\right)\right. \\
& \left.+\left(b_{2}+h\right) G_{2}\left(Q+R \mid k_{2}\right)-b_{1}\right]+\left(b_{2}+h\right) R^{\prime} .
\end{aligned}
$$

If $d^{2} R / d Q^{2}=0$, which is the case for $R=R^{*}$, we obtain

$$
\begin{aligned}
\frac{d^{2} E\left[T R C \mid k_{2}, d_{1}^{1}, d_{1}^{2}\right]}{d Q^{2}}= & \left(1+R^{\prime}\right)^{2}\left[\left(b_{1}-b_{2}\right) g_{2}^{1}\left(Q+R \mid k_{2}\right)\right. \\
& \left.+\left(b_{2}+h\right) g_{2}\left(Q+R \mid k_{2}\right)\right],
\end{aligned}
$$

which is non-negative for all $k_{2}$, because $\left(1+R^{\prime}\right)^{2} \geq$ $0, b_{1}>b_{2}$, and $g_{2}^{1}\left(Q+R \mid k_{2}\right)$ and $g_{2}\left(Q+R \mid k_{2}\right)$ are density functions. Consequently, E[TRC $\left.\mid k_{2}, d_{1}^{1}, d_{1}^{2}\right]$ is convex in $Q$ for a given set of $d_{1}^{1}, d_{1}^{2}$, and $k_{2}$.

Finally, we note that $E[T R C(Q)]=E_{K_{2}, D_{1}^{1}, D_{1}^{2}}[E[T R C \mid$ $\left.k_{2}, d_{1}^{1}, d_{1}^{2}\right]$. Consequently, since expectations can be written as the limits of Riemann-Stieltjes sums, and the positive-weighted sum of convex functions are convexsee, e.g. Heyman and Sobel (1984)—we conclude that $E[T R C(Q)]$ is convex in $Q$.

\section{References}

Benjaafar, S., Cooper, W.L., Mardan, S., 2007. Production-Inventory Systems with Imperfect Advance Demand Information and Updating, Working Paper, University of Minnesota.

Cohen, M.A., Kleindorfer, P.R., Lee, H.L., 1988. Service constrained (s,S) inventory systems with priority demand classes and lost sales. Management Science 34, 482-499.

DeCroix, G.A., Mookerjee, V.S., 1997. Purchasing demand information in a stochastic-demand inventory. European Journal of Operational Research 102, 36-57.

Dekker, R., Hill, R.M., Kleijn, M.J., Teunter, R.H., 2002. On the (S-1, S) lost sales inventory model with priority demand classes. Naval Research Logistics 49, 593-610.

Dellaert, N.P., Melo, M.T., 2003. Approximate solutions for a stochastic lot-sizing problem with partial customer-order information. European Journal of Operational Research 150, 163-180.

Deshpande, V., Cohen, M.A., Donohue, K., 2003. A threshold inventory rationing policy for service-differentiated demand classes. Management Science 49, 683-703.

Enders, P., Adan, I.J.B.F., Scheller-Wolf, A., Van Houtum, G.J., 2008. Inventory Rationing for a System with Lost-Sales and a Backordering Customer Class. Tepper Working Paper \#2008-E2, Tepper School of Business, Carnegie Mellon University, USA.

Evans, R.V., 1968. Sales and restocking policies in a single item inventory system. Management Science 14, 463-472.
Frank, K.C., Zhang, R.Q., Duenyas, I., 2003. Optimal policies for inventory systems with priority demand classes. Operations Research 51, 993-1002.

Gallego, G., Özer, Ö., 2001. Integrating replenishment decisions with advance demand information. Management Science 47, 1344-1360.

Gayon, J.P., Benjaafar, S., de Véricourt, F., 2008. Using imperfect advance demand information in production-inventory systems with multiple customer classes, Manufacturing \& Service Operations Management, to appear.

Ha, A.Y., 1997a. Stock-rationing policy for a make-to-stock production system with two priority classes and backordering. Naval Research Logistics 44, 457-472.

Ha, A.Y., 1997b. Inventory rationing in a make-to-stock production system with several demand classes and lost sales. Management Science 43, 1093-1103.

Hariharan, R., Zipkin, P., 1995. Customer-order information, leadtimes, and inventories. Management Science 41, 1599-1607.

Heyman, D., Sobel, M., 1984. Stochastic Models in Operations Research, vol. II. McGraw Hill, New York, NY.

Karaesmen, F., Buzacott, J.A., Dallery, Y., 2002. Integrating advance order information in make-to-stock production systems. IIE Transactions 34, 649-662.

Karaesmen, F., Liberopoulos, G., Dallery, Y., 2003. Production/inventory control with advance demand information. In: Shanthikumar, J.G., Yao, D.D., Zijm, W.H.M. (Eds.), Stochastic Modeling and Optimization of Manufacturing Systems and Supply Chains. International Series in Operations Research and Management Science, vol. 63. Kluwer Academic Publishers, Boston, MA, pp. 243-270.

Kranenburg, A.A., Van Houtum, G.J., 2007. Cost optimization in the (S-1, S) lost sales inventory model with multiple demand classes. OR Letters 35, 493-502.

Liberopoulos, G., Koukoumialos, S., 2008. On the effect of variability and uncertainty in advance demand information on the performance of a make-to-stock supplier. MIBES Transactions International Journal 2, 95-114.

Melchiors, P., Dekker, R., Kleijn, M.J., 2000. Inventory rationing in an (s,Q) inventory model with lost sales and two demand classes. Journal of the Operational Research Society 51, 111-122.

Moon, I., Kang, S., 1998. Rationing policies for some inventory systems. Journal of the Operational Research Society 49, 509-518.

Nahmias, S., Demmy, W.S., 1981. Operating characteristics of an inventory system with rationing. Management Science 27, 1236-1245.

Sobel, M.J., Zhang, R.Q., 2001. Inventory policies for systems with stochastic and deterministic demand. Operations Research 49, 157-162.

Tan, T., Güllü, R., Erkip, N., 2007. Modelling imperfect advance demand information and analysis of optimal inventory policies. European Journal of Operational Research 177, 897-923.

Teunter, R.H., Klein Haneveld, W.K., 1999. Reserving spare parts for critical demand. Research Report, Graduate School/Research Institute System, Organisations and Management (SOM), University of Groningen.

Thonemann, U.W., 2002. Improving supply-chain performance by sharing advance demand information. European Journal of Operational Research 142, 81-107.

Topkis, D.M., 1968. Optimal ordering and rationing policies in a nonstationary dynamic inventory model with $n$ demand classes. Management Science 15, 160-176.

Treharne, J.T., Sox, C.R., 2002. Adaptive inventory control for nonstationary demand and partial information. Management Science 48, 607-624.

Tsay, A.A., Nahmias, S., Agrawal, N., 1999. Modeling supply chain contracts: a review. In: Tayur, S., Ganeshan, R., Magazine, M. (Eds.), Quantitative Models for Supply Chain Management. Kluwer's International Series, Boston, MA.

Van Donselaar, K., Kopczak, L.R., Wouters, M., 2001. The use of advance demand information in a project-based supply chain. European Journal of Operations Research 130, 519-538.

Veinott Jr., A.F., 1965. Optimal policy in a dynamic, single product, nonstationary inventory model with several demand classes. Operations Research 13, 761-778.

de Véricourt, F., Karaesmen, F., Dallery, Y., 2002. Assessing the benefits of different stock-allocation policies for a make-to-stock production system. Manufacturing \& Service Operations Management 3. 105-121.

de Véricourt, F., Karaesmen, F., Dallery, Y., 2002. Optimal stock allocation for a capacitated supply system. Management Science 48 , 1486-1501.

Zhu, K., Thonemann, U.W., 2004. Modeling the benefits of sharing future demand information. Operations Research 52, 136-147. 